\title{
Hard X-ray Nanoprobe Characterization of Advanced Materials
}

\author{
Volker Rose*, Martin Holt**, Robert Winarski**, Jörg Maser** \\ * Advanced Photon Source, Argonne National Laboratory, Argonne, Illinois 60439 \\ ** Center for Nanoscale Materials, Argonne National Laboratory, Argonne, Illinois 60439
}

The Advanced Photon Source (APS) at Argonne National Laboratory is a unique high-energy x-ray source, which provides state-of-the-art hard $\mathrm{x}$-ray tools available to scientists and engineers worldwide. The mission of the APS encompasses solving key problems in energy, environment, technology development, and human health. Through x-ray imaging, we can illuminate complex hierarchical structures from the molecular level to the macroscopic level, and study how they change in time and in response to stimuli. Hard x-rays are also especially suited to the study of real materials under realistic conditions and in realtime. In this talk we will focus on the new Hard X-Ray Nanoprobe $(\mathrm{HXN})$ at sector 26 of the APS, which is one of the world's most powerful x-ray microscopes [1,2]. It has been designed to study novel nanoscale materials and devices in previously unavailable detail and is particularly well suited for the study of buried structures in real environments and for dynamics. This next-generation hard x-ray microscopy and imaging system provides x-ray fluorescence, x-ray diffraction, coherent diffraction, transmission imaging, and tomography at a spatial resolution of $30 \mathrm{~nm}$ or better. The combination of diffraction, fluorescence, and transmission contrast in a single tool provides unique characterization capabilities for nanoscale imaging. In this overview talk, the basic principles of the instrument are discussed, accompanied by a presentation of recent results ranging from apoptosis in glioblastoma multiforme [3] to elemental mapping of geopolymers [4].

\section{References}

[1] J. Maser, R. Winarski, M. Holt, D. Shu, C. Benson, B. Tieman, C. Preissner, A. Smolyanitskiy, B. Lai, S. Vogt, G. Wiemerslage, G. B. Stephenson, in Proceedings of the 8th International Conference on X-ray Microscopy; Aoki, S., Kagoshima, Y., Suzuki, Y., Eds.; IPAP: Himeji, Japan, (2005) 26-29.

[2] D. Shu, J. Maser, M. Holt, R. Winarski, C. Preissner, A. Smolyanitskiy, B. Lai, S. Vogt, G. B. Stephenson, AIP Conf. Proc. 879 (2007) 1321.

[3] E.A. Rozhkova, I. Ulasov, B. Lai, N.M. Dimitrijevic, M.S. Lesniak, T. Rajh, Nano Lett. 9 (2009) 3337.

[4] J.L. Provis, V. Rose, S.A. Bernal, J.S.J. van Deventer, Langmuir 25 (2009) 11897.

$\uparrow$ This work was supported by the U. S. Department of Energy, Office of Science, Office of Basic Energy Sciences, under Contract No. DE-AC02-06CH11357. 


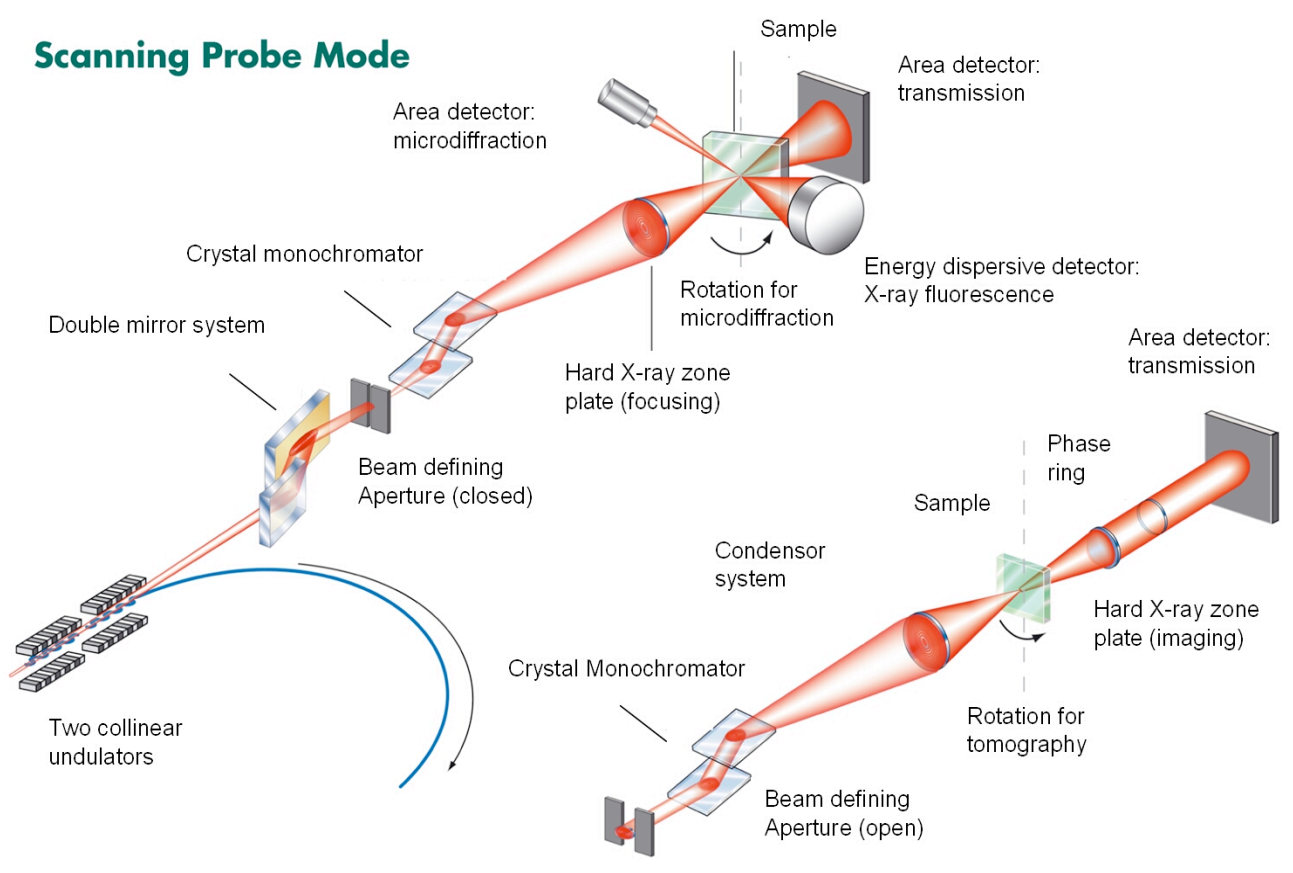

Full-Field Transmission Mode

FIG. 1. The HXN can be operated in one of two different modes depending on the scientific problem. It allows characterization of nanoscale materials and devices at highest spatial resolution.
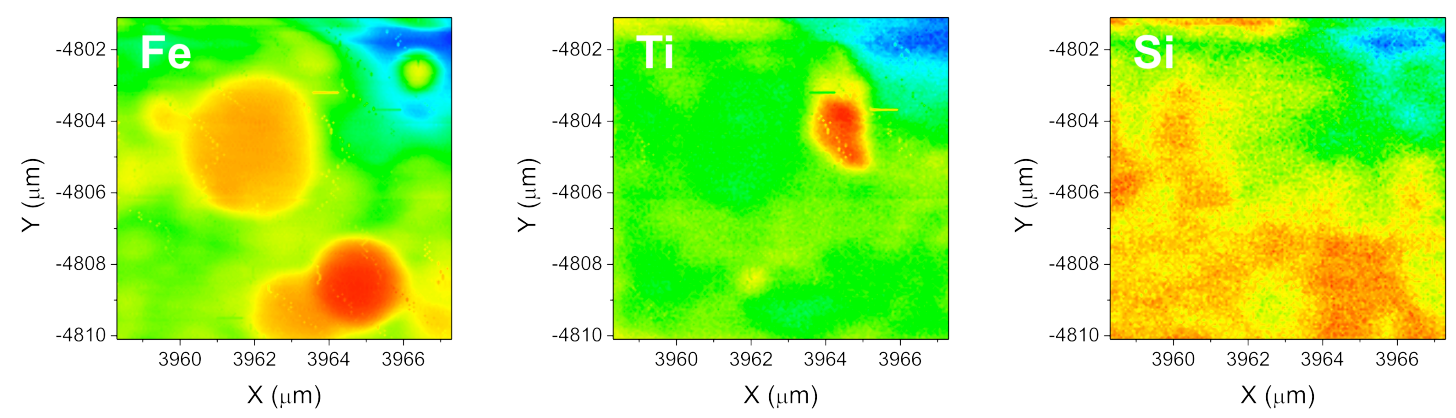

FIG. 2. Iron, titanium and silicon distribution in hydroxide-activated geopolymer imaged by x-ray fluorescence. The exceptional spatial resolution and penetration power of the HXN enables the study of poorly understood heterogeneity and complex chemistry of geopolymers. 